\title{
Large Corneal Grafts can be Successful
}

\author{
C. M. KIRKNESS, L. A. FICKER, N. S. C. RICE, A. DMcG. STEELE \\ London
}

\begin{abstract}
Summary
Seventeen grafts of $10 \mathrm{~mm}$ in diameter or larger have been performed on 16 eyes of 15 patients. The major indications for surgery were infections or perforations or both. Follow-up has ranged from 8 - 54 months (mean 26.4). The 4 year survival probability was 0.64 . Although the procedure was successful in saving all but one eye and restored useful vision in the majority, complications including cataract, glaucoma, graft rejection episodes and infections were encountered. The management of these complications is described. At final review, 13 eyes had clear grafts including those in whom regraft had been performed. These results have only been achieved by close co-operation between patients and the medical team responsible for their care.
\end{abstract}

Traditional wisdom cautions against performing large corneal grafts because of the risks of allograft rejection. This is likely because of the large antigen load provided, particularly so when one considers that the Langerhans cells are only found in the peripheral cornea, ${ }^{1}$ and the proximity to the vascular tissue of the limbus. ${ }^{2}$ When corneas are transplanted into densely vascularised tissue it is known that the rejection rate closely approximates to rejection rates in other perfused tissues. ${ }^{2}$ An additional problem anticipated would be destruction of or damage to the drainage angle of the anterior chamber when the graft size exceeded $10 \mathrm{~mm}$ in diameter. There are, however, instances when large grafts may become necessary when the eye is to be saved and visual potential preserved. Young and Watson have described the use of large lamellar grafts when there is severe sclero-keratitis. ${ }^{3} \mathrm{Tico}^{4}$ reported whole $(10 \mathrm{~mm})$ corneal transplants and $\mathrm{Du},{ }^{5}$ and Casey ${ }^{6}$ have described the technique for keratoplasty in infected eyes without specifically dealing with the question of large penetrating grafts although some examples are quoted of whole corneal transplants. Recently, Guildford ${ }^{7}$ has produced disappointing results for such grafts. This paper aims to identify possible indications for large penetrating grafts and to describe their management and complications.

\section{Patients and methods}

Corneal grafts in excess of $9.5 \mathrm{~mm}$ in diamter have been designated as large grafts. This definition is appropriate since the wound edge will be a maximu'm of $1 \mathrm{~mm}$ inside the limbus and may extend beyond it; and the sutures will pass not only through the limbis but may invade the angle of the anterior chamber. The patients described form a consecutive series under the care of the surgeons of Corneal Clinic, Moorfields Eye Hospital, but the surgery was performed by one surgeon $(\mathrm{CMK})$. Only patients with a minimum follow-up of 6 months are included. None of the donor material nor recipients were tissue matched.

\section{Surgical technique}

The surgical technique was based upon gen- 
eral principles of corneal grafting but several points were of considerable importance. Since many of the grafts were performed for perforations, particular care was taken in preparing the host bed.

(1) The trephine size was chosen so that the entire wound edge was in healthy tissue, even when this meant the graft extending beyond the limbus.

(2) If, in the case of grafts extending beyond the limbus, it was possible to preserve some limbal tissue, and perform either a sector keratoplasty or to place the graft slightly eccentrically, this was done.

(3) Having chosen the appropriate trephine size for the host, the host bed was gently marked but no attempt to cut host tissue with the trephine was made. This was done with either a diamond blade or razor fragment free-hand using the trephine mark as a guide. Excess pressure with trephine can distort the tissues excessively.

(4) The size of the host bed was carefully measured (the cord length) and an appropriate donor size was chosen. Because of the low intraocular pressure of the host, there was often a disparity of $1 \mathrm{~mm}$ or more between the nominal trephine size used for host and donor, the donor by necessity being larger. ${ }^{8}$ Where possible a whole, moist chamber-stored eye was used as donor on the basis that it gave greater freedom in cutting an appropriate donor button.

(5) Visco-elastic delamination of the underlying tissue using healon or hydroxypropylmethyl cellulose $2 \%$ prevented damage to the underlying structures and assisted gonioplasty in eyes which were perforated.

(6) Anterior cyclitic membranes were removed and posterior synechiae were broken but no attempt was made to remove the lens unless it was intumescent or subluxed.

(7) When the eyes were aphakic an interior vitrectomy was performed to prevent incarceration of gel, and to remove any inflammatory or infected material.

(8) Since all eyes in the series underwent keratoplasty à chaud, interrupted $10 / 0$ nylon sutures were used.

(9) Where there was bare sclera, whether host or donor, it was covered with host con- junctiva which was sutured with $8 / 0$ virgin silk.

(10) Where the indication for surgery included active suppuration, specimens of all excised tissue were sent for microbiological examination.

Post-operatively, systemic steroids were not used routinely, but frequent application of topical steroids was used, usually to begin with Dexamethasone $0.1 \%$ as frequently as every hour. The dosage was gradually reduced, but many patients received longterm therapy. Antibiotics appropriate to the condition were also used.

In addition, any co-existent condition was treated as indicated, e.g. severe dry eye was treated with frequent artificial tear drops and if that was insufficient, by punctal occlusion. In very large grafts, where a considerable amount of limbus had been excised, lid taping was employed when re-epithelialisation was slow. Raised intraocular pressure was regarded as abnormal above $21 \mathrm{mmHg}$. Patients were seen at very frequent intervals during the first year after surgery and were instructed to contact the hospital immediately if there were any evidence of rejection. Rejection was diagnosed on the basis of a ciliary flush, the presence of flare and cells in the anterior chamber, in a previously quiet eye, keratitic precipitates on the donor endothelium, but not necessarily a Khodadoust line.

\section{Results}

Seventeen grafts larger than $9.5 \mathrm{~mm}$ in diameter are reported, in 16 eyes of 15 patients. Included in this figure is one autograft of $10 \mathrm{~mm}$ in diameter. Patients ages ranged from 16 at the time of surgery to 74 years, mean 47.9 years. Graft sizes ranged from $10 \mathrm{~mm}$ to $15 \mathrm{~mm}$ in diameter, median 10.5, mean 11.3. (Fig. 1). Follow-up ranged from 8 months to 54 months, median 22 months, mean 26.4 months. The indications for keratoplasty and the nature of any underlying relevant pathology are shown in Table I. For those cases where there was suppuration, the infecting organisms are shown in Table II. In 3 cases despite rigorous attempts, no organisms were identified. 
Table I CF, counts fingers; CSG, chronic simple glaucoma; HM, hand motion; HSK, herpes simplex keratitis; NPL, no perception of light; PBK, pseudophakic bullous keratopathy; Perf, perforation; SK suppurative keratitis

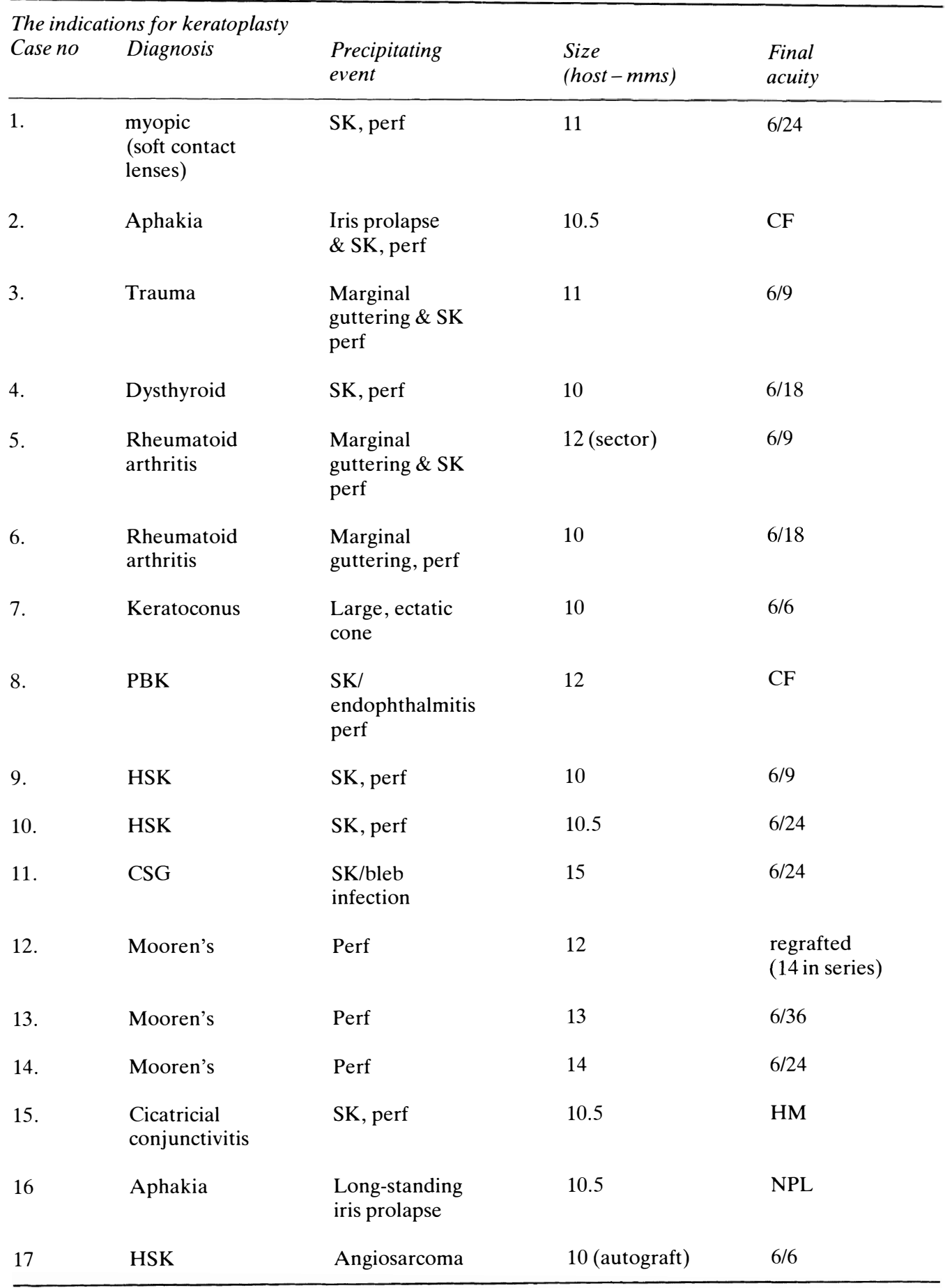


Table II The infecting organism in suppurative cases. The case numbers are consistent with Table I

\begin{tabular}{ll}
\hline Case no & Infecting organism \\
\hline 1 & pseudomonas sp \\
2 & Aspergillus terreus. \\
3 & No organism identified \\
4 & Moraxella \\
5 & Streptococcus pneumoniae, moraxella, \\
& Haemophillus influenzae, \\
& staphylococcus epdermidis. \\
8 & No organism identified \\
9 & No organism identified \\
10 & pseudomonas sp \\
11 & Streptococcus pneumoniae \\
12 & Staphylococcus aureus
\end{tabular}

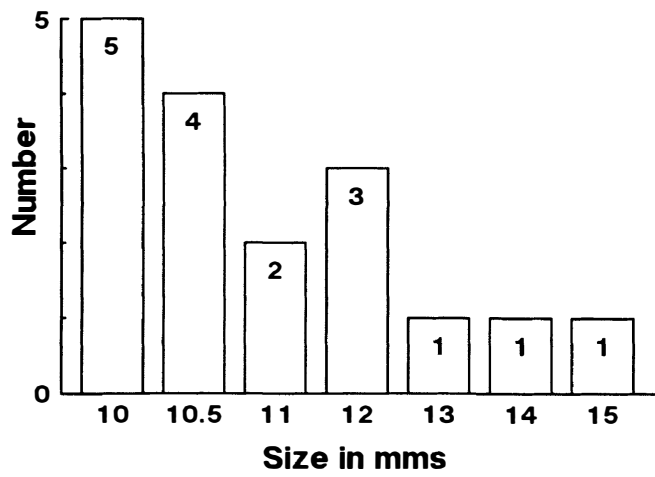

Fig. 1. The size of the host bed as defined by nominal trephine size.

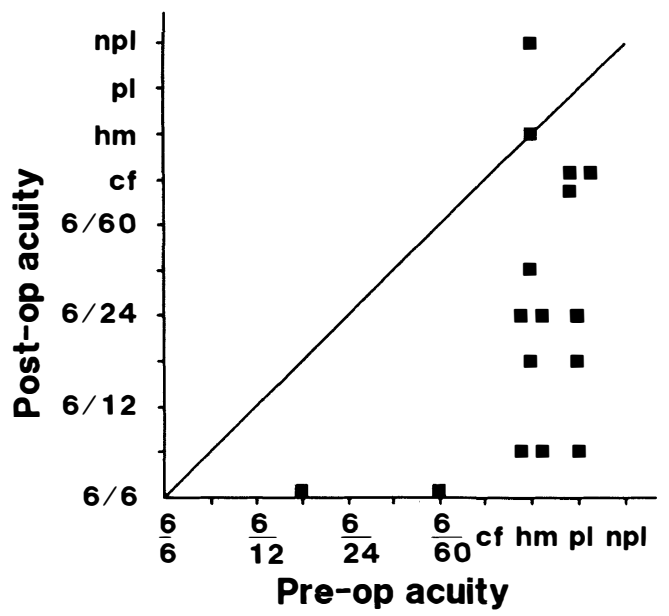

Fig. 2. A scattergram showing the visual acuity at final review in the 16 eyes compared with immediately pre-operative acuities.
Visual acuity at final review, was $6 / 18$ or better in 7 eyes, $(41.3 \%)$ and less than $6 / 60$ in 5 eyes, (29.4\%) (Fig. 2.) In these latter cases the poor vision was due to extensive myopic chorioretinal degeneration (1, no 2$)$, cystoid macular oedema (1, no 8$)$, a new infective episode $(2$, nos. 15,16$)$ and graft oedema (1, no 11 - this patient is currently awaiting

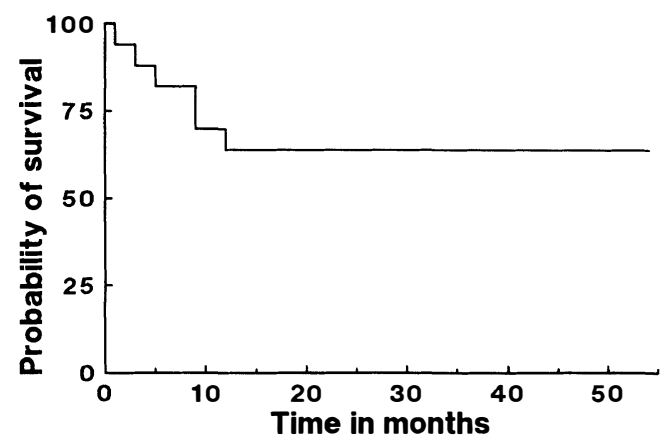

Fig. 3. The survival curve for all grafts plotted according to the methods of Kaplan \& Meier, ${ }^{9}$ i.e. the probability of graft survival is plotted against time.

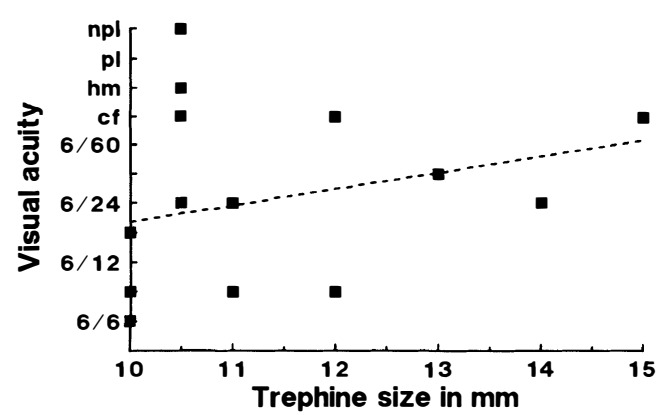

Fig. 4. Scattergram of final acuity against size of trephine used.

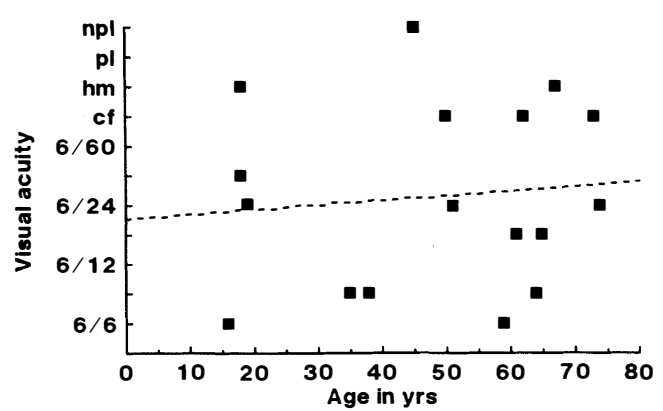

Fig. 5. Scattergram of final acuity against age of patient at time of surgery. 


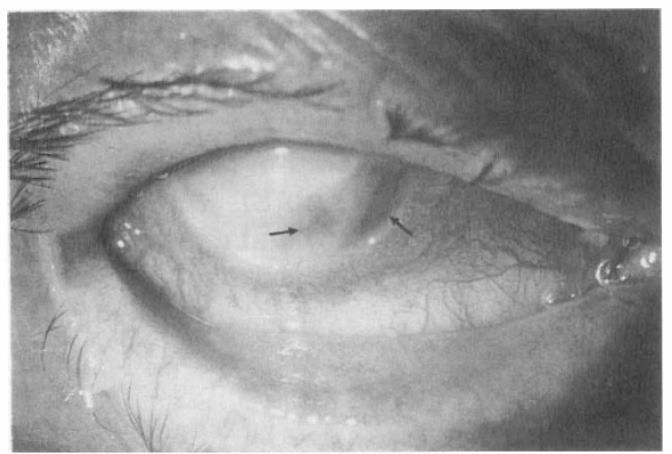

Fig. 6. A patient (no 5) with rheumatoid arthritis and peripheral corneal guttering which had become infected with 4 separate organisms. There is dense infiltrate in the base of the gutter and there is a hypopyon. The gutter perforated in several places, (shown with arrows).

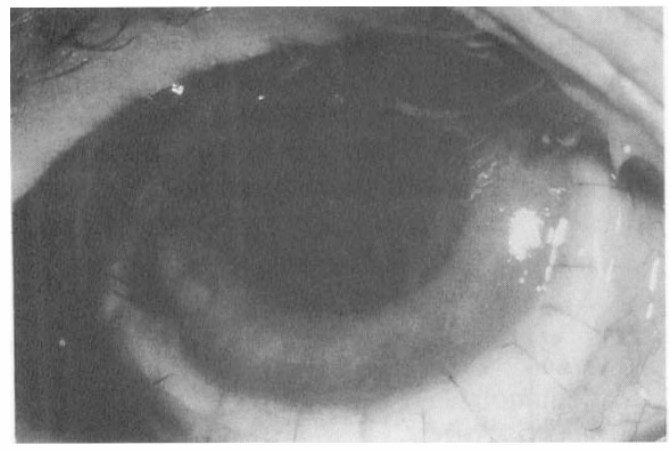

Fig. 7. The same eye 3 months later. Visual acuity is $6 / 9$.

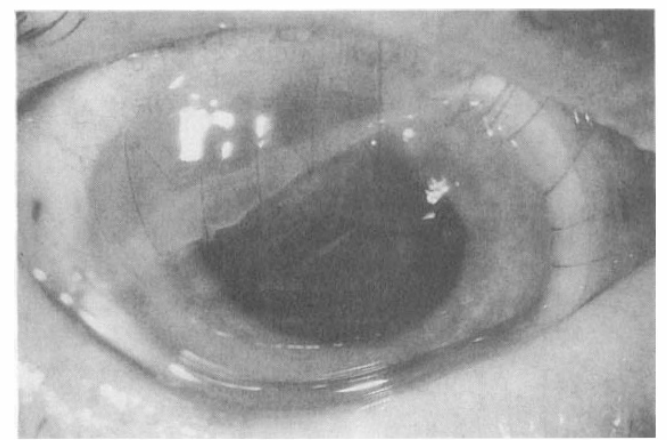

Fig. 8. (Same as figure 7) This view shows the straight edged part of the sector keratoplasty, which has enabled preservation of a quadrant of limbus which, in turn, helped re-epithelialisation. The straight edge has to be cut free-hand in both donor and host and the running suture helps to achieve water-tight closure. regraft). The overall graft survival is shown in Figure 3. This is plotted according to the methods of Kaplan and Meier. ${ }^{9}$ There is a $64 \%$ probability of graft survival at four years. Figure 2 shows a scattergram of final visual acuity (including regrafted eyes) against pre-operative acuity. Figures 4 and 5 show scattergrams of final acuity against graft size and age. In neither case is there a strong correlation between these parameters. It should be noted that although a regression line is drawn the regression coefficient cannot be accurately calculated because the scaling of the visual acuity axis is neither linear nor regular. Figures $6-8$ show a typical patient before and after surgery.

\section{Complications}

A number of important complications were encountered. The numbers and relationships are shown in the Venn diagram in Figure 9. There were no wound related problems, nor did any infected eye subsequently develop endophthalmitis.

Cataract developed in 5 eyes following keratoplasty between 6 months and 12 months later, and was successfully removed in 4 instances without prejudicing graft survival. In the remaining case the cataract is not severe enough to warrant extraction. In two out of the four cases when extraction was undertaken, surgery was combined with an intraocular implant.

Raised intraocular pressure (IOP) developed in five patients all of whom were

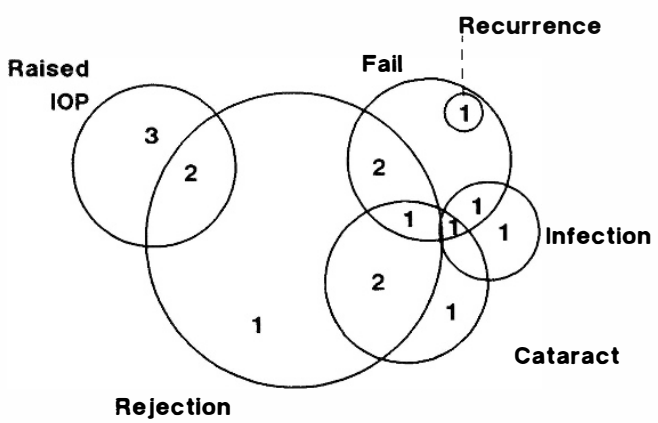

Fig. 9 Venn diagram to show the relationship between the complications related to individual eyes, demonstrating that several eyes suffered more than one complication though not necessarily simultaneously. 
aphakic. In two cases, the IOP was a problem prior to surgery but it became worse following keratoplasty. Three cases required the use of silicone drainage tubing to control intraocular pressure adequately, one was controlled following trabeculectomy and the other was managed with medical therapy. Corneal graft reactions were common, being seen in 8 eyes but they were successfully reversed in 5 eyes. Only 3 grafts failed as a result of rejection. One eye (no 9) had repeated episodes of corneal graft rejections but in each case they have been diagnosed early and treatment with topical steroids has been successful in reversing the rejection.

In 3 eyes, further episodes of infection recurred unrelated to the original event leading to keratoplasty, (i.e. there was a different infecting organism in two of the cases which had been grafted for suppuration and one case which was grafted for iris and uveal prolapse subsequently developed a suppurative keratitis). In all these cases there was considerable ocular surface abnormality, which predisposed to infection.

One eye was regrafted after Mooren's ulceration had recurred and led to perforation of the eye after minor trauma. In all, six grafts failed; four have been regrafted, three of these are clear with a follow-up of 6 months to 38 months (mean 15 months), and one failed following a further episode of infection (no 15). One case awaits regrafting after a recent rejection episode. Thirteen eyes therefore have clear grafts or regrafts at the time of review.

\section{Discussion}

In 10 eyes out of the 17 , the major indication for surgery was corneal infection which had led to perforation or was uncontrolled on medical treatment. Only one case was associated with pre-operative endophthalmitis. Although the place of keratoplasty in suppurative keratitis has been described, ${ }^{4-7,10}$ accepted guidelines have not been laid out. In his book, Casey ${ }^{7}$ recommends that eyes which are perforated and infected should be treated with systemic steroids for 48 hours prior to keratoplasty in order to suppress inflammatory activity. There is a suggestion from our cases and others reported separately by Kirkness ${ }^{11}$ that the longer an eye is perforated the greater the likelihood that it will sustain damage to the angle and be predisposed to post-keratoplasty glaucoma. Once a decision has been made that keratoplasty is indicated the authors feel that there is no advantage in postponing surgery.

At surgery for infected cases, portions of all excised tissue and intraocular fluids should be sent directly for microbiological investigation. This allows a logical reappraisal of antimicrobial therapy postoperatively. ${ }^{12}$

Few authors have addressed themselves specifically to the question of the survival of large grafts. Tico et $a l^{4}$ reported a series of eyes undergoing $10 \mathrm{~mm}$ corneal grafts but, after a short follow-up, only 8 eyes (including regrafts) out of 14 eyes saw better than $3 / 60$. Du et al ${ }^{5}$ included 15 grafts greater than $8 \mathrm{~mm}$ in diameter in their very large series of 100 keratoplasties for suppurative keratitis. Of these 6 were $10 \mathrm{~mm}$ in diameter or larger. At one year following surgery, only 4 grafts out of the group of 15 were clear. VolkerDieben ${ }^{13}$ suggested that grafts larger than 8.1 $\mathrm{mm}$ in diameter had a reduced prognosis compared to smaller grafts, but she did not state the range of size of the grafts. In vascularised corneas the 3 year survival was approximately $50 \%$ for tissue-matched corneas.

The survival curve for the present series of large corneal grafts shows a probability of corneal graft survival of .64 at 4 years. It is interesting to note that the failures all occurred in the first year after keratoplasty. The survival of these grafts is similar to that seen in first grafts for herpes simplex keratitis. ${ }^{14}$ This result is especially encouraging in view of the fact that the grafts were performed primarily to save the eye and to restore ocular integrity. Where graft failure had occurred and regrafting was attempted, the results have also been encouraging and suggest that even if a large graft eventually fails, a second graft may be undertaken with a view to restoring useful vision.

Although few eyes achieved 6/6 visual acuity, in many instances the premorbid visual potential was reduced and normal acuity 
could not be expected due to other, unrelated pathological changes.

Development of cataract was a common complication in those patients who were phakic at the time of keratoplasty. Of the 9 eyes which were phakic at the completion of the graft surgery, 5 developed significant lenticular opacity. Four cataracts have been successfully removed without prejudice to the graft endothelium. Lemp ${ }^{15}$ suggested that intraocular surgery was prejudicial to the corneal graft, but it is likely that the introduction of visco-elastic surgery has greatly reduced this risk. It is the authors' contention that cataract surgery should be avoided at the time of keratoplasty unless the lens is intumescent or dislocated because of the technical difficulties involved in removing the lens by an open sky technique when a large host bed has been prepared.

When the graft-host wound extends posterior to the limbus it is likely that anterior ciliary arteries are cut and as a result there will be some ciliary body hypo-perfusion or ischaemia. This in turn will hasten the development of lens opacity. Glaucoma was also a frequent complication which would be expected from the number of aphakic eyes. ${ }^{16}$ In addition some alteration in outflow facility should be expected either from mechanical compression of the angle by the sutures passing through the trabecular meshwork and from the development of fine peripheral synechiae which are inevitable in graft wounds immediately overlying the angle of the anterior chamber. In several instances the angle was entirely replaced and the scleral spur was dissected from the excised host tissue through $360^{\circ}$. The resulting aqueous dynamics can only be guessed at, but again, ciliary body hypo-perfusion may explain why raised pressure was not more common. Tico ${ }^{4}$ found a similar percentage of cases developing glaucoma. It is also probable that a number of small clefts persisted between the ciliary body and graft allowing drainage of aqueous to the suprachoroidal space and hence to the uveo-scleral outflow. When intractable glaucoma did develop, the use of silicone drainage tubing was invaluable in controlling IOP. ${ }^{17}$

Graft rejection episodes occurred fre- quently but because the patients were alert to the fact, and because they presented early for treatment many grafts were preserved. This emphasises the need for constant surveillance and the need for the patients to have access to an ophthalmologist who can diagnose and treat allograft reactions promptly. ${ }^{18}$ The large size of the grafts did not appear to make adequate treatment of the rejection more difficult. Lechler ${ }^{19}$ suggested that a minimum number of dendritic cells was required to trigger $\mathrm{T}$-helper cells and destroy renal transplants in rats. This figure lies between 10,000 and 50,000 . Using Rodriques ${ }^{20}$ figures of $15-$ 20 dendritic cells $/ \mathrm{mm}^{2}$ in the peripheral third of clear cornea, there should be insufficient dendritic cells in the transplant for triggering even in a graft $10 \mathrm{~mm}$ in diameter or larger. Large corneal grafts are therefore unlikely to be more at risk of rejection because of the numbers of dendritic cells in the donor. The likelihood is that they are no more at risk than any graft in a vascular cornea. The high dose topical steroids may therefore have had a beneficial effect.

Patients with severe ocular surface disease are at greater risk of developing suppurative keratitis. ${ }^{21}$ It is disappointing that this fact is highlighted in this series. The predisposition is increased by the additional need for longterm topical steroids. One patient with bilateral juvenile Mooren's ulceration showed recurrence of the ulcer in one eye despite systemic steroids and cyclosporin therapy. The ulcer recurred within 6 months and following perforation after minor trauma, the regrafted eye has remained free of the disease for 12 months. Young and Watson ${ }^{3}$ and Mondino ${ }^{22}$ have described how grafting during the acute inflammatory event may have disappointing results from the point of view of graft survival. Nevertheless, when an eye has perforated, keratoplasty may be necessary to save the eye. In contrast to many of these problems, the patient who underwent the autograft $10 \mathrm{~mm}$ in diameter experienced no complications related to the keratoplasty, suggesting that the degree of inflammation in most of these eyes was an adverse factor. The results of this series suggest that large grafts did not carry the very poor prognosis that is feared by many surgeons. They have, how- 
ever, only been achieved by close co-operation between patient and surgeons and by meticulous attention to medical and surgical therapy.

\section{References}

${ }^{1}$ Rowden G: Expression of Ia antigens in Langerhans cells in mice, gunea pigs and man. J Invest Dermatol 1980; 75: 22-31.

${ }^{2}$ Braude, LS and Chandler, JW: Corneal allograft rejection. The role of the major histocompatibility complex. Surv Ophthalmol 1983, 27: 290-305.

3 Young RD and Watson PG: Light and electron microscopy of corneal melting syndrome (Mooren's ulcer). Br J Ophthalmol 1982, 66: 341-56.

${ }^{4}$ Tico V and Ben Sira I: Total keratoplasty. Arch Ophthalmol 1973, 90: 104-6.

${ }^{5} \mathrm{Du}$ NZ, Chen JO, Gong XM et al.: Therapeutic keratoplasty in the management of purulent ulceration. Report of 100 cases. Jap J Ophthalmol 1979, 23: 412-20.

${ }^{6}$ Casey TA and Mayer DJ: Corneal grafting. Principles and practice. WB Saunders Co, Philadelphia, 1984 Ch 21; 253-260.

${ }^{7}$ Guildford JH, Alfonso E, Roussel TJ et al.: Large therapeutic keratoplasty in microbial keratitis. Invest Ophthalmol Vis Sci 1988, 29: 6.

${ }^{8}$ Olson RJ: Variation on corneal graft size related to trephine technique. Arch Ophthalmol 1979, 97: 1323-5.

${ }^{9}$ Kaplan E and Meier, P: Nonparametric estimation from incomplete observations. $\mathrm{J} \mathrm{Am}$ Statist Ass 1958, 53: 457-81.

${ }^{10}$ Hill JC: Use of penetrating keratoplasty in acute bacterial keratitis. Br J Ophthalmol 1986, 70: $502-6$.
${ }^{11}$ Kirkness CM and Moshegov C: Postkeratoplasty glaucoma. Eye 1988, 2: (Suppl): 519-26.

12 Jones DB: Decision making in microbial keratitis. Ophthalmology 1981, 88: 814-20.

${ }^{13}$ Volker-Dieben HJ, D'Amaro, Kok-van Alphen CC et al:: The interactions of HLA-Dr, donor graft size and corneal vascularisation of the recipient on graft survival. Doc Ophthalmol 1984, 57: 67-77.

${ }^{14}$ Ficker LA, Kirkness CM, Rice NSC, Steele ADMcG. The long term prognosis for graft survival in herpes simplex keratitis. Eye 1988, 2: $400-8$.

${ }^{15}$ Lemp MA, Pfister RR, Dohlman CH: The effect of intraocular surgery on clear grafts. Am J Ophthalmol 1970, 70: 719-21.

${ }^{16}$ Irvine AR and Kaufman HE: Intraocular pressure following penetrating keratoplasty. Am J Ophthalmol 1969, 68: 835-44.

${ }^{17}$ Kirkness CM: Penetrating keratoplasty, glaucoma and silicone drainage tubing. Dev Ophthalmol (Karger, Basel) 1987, 14: 165-9.

18 Morris R and Kirkness CM: Emergency presentation of corneal graft patients. Eye 1988, 2: 71-6.

${ }^{19}$ Lechler RJ and Batchler JR: Restoration of immunogenicity to passenger cell depleted kidney allografts by the addition of donor strain dendritic cells. J Exp Med 1982, 155: $31-41$.

${ }^{20}$ Rodriques MM, Rowden G, Hacket $\mathrm{J}$ et al.: Langerhans cells in the normal conjunctiva and peripheral cornea of selected species. Invest Ophthalmol Vis Sci 1981, 21: 759-65.

${ }^{21}$ Kenyon KR: Morphology and pathologic responses of the cornea to disease. In: The cornea, 2nd edition. Smolin G, Thoft RA. eds Little, Brown \& Co. Boston, 1987. Ch 2, p 63-98.

${ }^{22}$ Brown SI and Mondino BJ: Penetrating keratoplasty in Mooren's ulcer. $A m$ J Ophthalmol 1980; 89: 255-8. 